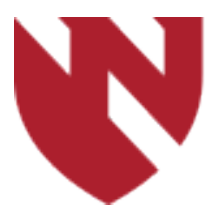

December 2019

\title{
Without Name DAH
}

Ian Cormier

University of Nebraska Medical Center

Edin Pujagic

University of Nebraska Medical Center

Neil Bhogal

University of Nebraska Medical Center

Michael Smith

University of Nebraska Medical Center

Tell us how you used this information in this short survey.

Follow this and additional works at: https://digitalcommons.unmc.edu/gmerj

Part of the Higher Education Commons, and the Medicine and Health Sciences Commons

\section{Recommended Citation}

Cormier, I., Pujagic, E., Bhogal, N., , Smith, M. Without Name DAH. Graduate Medical Education Research Journal. 2019 Dec 13; 1(1).

https://digitalcommons.unmc.edu/gmerj/vol1/iss1/22

This Conference Proceeding is brought to you for free and open access by DigitalCommons@UNMC. It has been accepted for inclusion in Graduate Medical Education Research Journal by an authorized editor of DigitalCommons@UNMC.For more information, please contact digitalcommons@unmc.edu. 
Without Name DAH

Creative Commons License

(1) $\odot \Theta \Theta$

This work is licensed under a Creative Commons Attribution-Noncommercial-No Derivative Works 4.0 License. 


\section{Genotype and Phenotype Correlation in Patients with Comorbid Epilepsy and Intellectual Disability} Mohamed Taha, Jill Clayton-Smith

Mentor: Jill Clayton-Smith

Program: Neurology

Epilepsy and intellectual disability comorbidity is a common condition with great medical and psychological impact on a patient's life and their family members.

We sought to investigate the genotype and phenotype of patients with epilepsy and intellectual disability comorbidity who had negative karyotyping and microarrays results, to find any statistical correlations of positive findings with specific clinical features, and to calculate the diagnostic rates of targeted exome sequencing panel and whole exome sequencing.
In 80 patients with the comorbid condition, recruited by the Manchester Centre of Genomic Medicine, we analyzed the relevant clinical information, collected data from 76 patients who were tested by a targeted exome sequencing panel assembled to diagnose this condition, and gathered the data of 16 patients who subsequently went on to be tested by whole exome sequencing.

The overall detection rate of pathogenic and likely pathogenic variants in genes associated with epilepsy and intellectual disability comorbidity was $24 \%$. There was a $17 \%$ detection rate by the targeted exome sequencing panel $(13 / 76)$, and $33 \%$ by whole exome sequencing $(5 / 15)$. There was no significant statistical correlation identified between genotype and phenotype variables. The STXBP1 gene was the gene most frequently identified to carry a disease causing variant, seen in 5 patients.

This study demonstrated the considerable utility of the use of targeted exome sequencing as well as whole exome sequencing in this condition for detection of causative genetic variants. The absence of statistical correlation between the chances of finding a pathogenic result and a specific phenotype, suggests that the clinical presentations within this group are not always distinctive or that the use of a much larger study group is needed to improve the statistical power.

https://doi.org/10.32873/unmc.dc.gmerj.1.1.020

\section{Correlation of the Multi-Biomarker Disease Activity Score with Rheumatoid Arthritis Disease Activity Measures: A Systematic Review and Meta-Analysis \\ Tate Johnson, Kyle Register, Cynthia Schmidt, James O'Dell, Ted Mikuls, Kaleb Michaud, Bryant England}

Mentor: Bryant England

Program: Internal Medicine

Background: There are conflicting reports on the validity of the multi-biomarker disease activity (MBDA) score to assess rheumatoid arthritis (RA) disease activity. We performed a systematic review of the MBDA and metaanalysis of the correlation between the MBDA and other RA disease activity measures.

Methods: A systematic review was performed, searching MEDLINE, EMBASE, Scopus, Google Scholar, and the Cochrane Library from inception to March 7, 2017. Study details, MBDA performance, and study quality were assessed by independent reviewers. Correlations of the MBDA with composite RA disease activity measures were pooled using random-effects meta-analyses.

Results: Twenty-two studies were identified in the systematic review, of which $8(n=3,242$ assays) reported correlations of the MBDA with RA disease activity measures. Pooling results from these eight studies in the metaanalysis, the MBDA demonstrated modest correlations with DAS28-CRP $(r=0.41,95 \%$ CI 0.36-0.46) and DAS28-ESR $(r=0.48$, $95 \%$ CI $0.38-0.58)$ with weaker correlations observed with SDAI $(\mathrm{r}=0.35,95 \%$ CI 0.26 $0.43)$, CDAI ( $\mathrm{r}=0.26,95 \%$ CI $0.19-0.33$ ), and RAPID3 ( $\mathrm{r}=0.23,95 \%$ CI $0.19-0.27)$. Correlations between change in MBDA and change in disease activity measures ranged from $r=0.53$ (DAS28-ESR) to $r=0.26$ (CDAI).

Conclusion: The MBDA demonstrates moderate convergent validity with DAS28CRP and DAS28-ESR, but weaker correlations with SDAI, CDAI, and RAPID3. While it appears to complement existing RA disease activity measures, further assessment of the MBDA's performance characteristics is warranted.

https://doi.org/10.32873/unmc.dc.gmerj.1.1.021

\section{Without Name DAH}

Ian Cormier, Edin Pujagic, Neil Bhogal, Michael Smith

\section{Mentor: Michael Smith \\ Program: Internal Medicine}

Hantavirus Cardiopulmonary Syndrome (HCPS) is a relatively uncommon zoonotic viral illness classically associated with the southwestern United States. It is important for clinicians to include HCPS on the differential for diffuse alveolar hemorrhage (DAH) and acute respiratory distress syndrome (ARDS), as HCPS carries a high mortality rate and commonly goes unrecognized.

A middle-aged patient presented with fever and cough and was found to be hypotensive and thrombocytopenia. Despite being started on broad spectrum antibiotics, the patient's respiratory status rapidly declined, requiring intubation and ICU level of care. Chest radiographs revealed diffuse pulmonary infiltrates and computed tomography of the chest revealed severe bilateral pulmonary consolidation and edema resembling ARDS. A bronchoalveolar lavage revealed diffuse alveolar hemorrhage. All infectious and rheumatologic workup was negative except for a significantly elevated Hantavirus IgM which was reflexed to a state public health laboratory. In the United States, HCPS is most commonly caused by the Sin Nombre virus (SNV), a member of the Hantavirus genus transmitted primarily by the deer mouse. This host has an extensive habitat across much of North America but the majority of confirmed HCPS cases occur in the Southwestern U.S. All clinically important Hantaviruses are transmitted by aerosolized droplets from urine, feces or saliva of rodents. The cardiopulmonary phase of HCPS is characterized by capillary leak into the alveoli, causing bilateral diffuse interstitial edema and pulmonary hemorrhage that may resemble ARDS on imaging. Patients with suspected HCPS should be screened for Hantavirus IgM and IgG. Positive IgM samples from U.S. residents are sent to a Public Health Laboratory for SNV-specific antibody testing.

https://doi.org/10.32873/unmc.dc.gmerj.1.1.022 